\title{
Phase II Study of Cetuximab in Combination with Docetaxel in Patients with Recurrent and/or Metastatic Squamous Cell Carcinoma of the Head and Neck after Platinum-Containing Therapy: A Multicenter Study of the Arbeitsgemeinschaft Internistische Onkologie
}

\author{
M. Knoedler ${ }^{\text {a }} \quad$ T.C. Gauler ${ }^{b} \quad$ V. Gruenwald ${ }^{c} \quad$ A. Matzdorff ${ }^{d} \quad$ M. Schroeder
}
A. Dietz ${ }^{f}$ W.-O. Jordan ${ }^{g}$
D. Arnold ${ }^{\mathrm{h}}$
B. Hennemann ${ }^{i}$
C. Hofele ${ }^{j}$
F. Weissingerk
W. Eberhardt ${ }^{\mathrm{b}} \quad$ U. Keilholz ${ }^{\mathrm{a}}$

\begin{abstract}
a Department of Hematology and Medical Oncology, Charité Berlin, Berlin, b'Internal Medicine (Tumor Research), University Hospital Essen, Essen, 'Department of Oncology, Medizinische Hochschule Hannover, Hannover, dDepartment of Oncology, Caritas Klinik St. Theresia Saarbrücken, Saarbrücken, ${ }^{e}$ Department of Oncology, Hospital St. Johannes, Duisburg, ${ }^{f}$ Head and Neck Surgery, University Hospital Leipzig, Leipzig, ${ }^{9}$ Department of

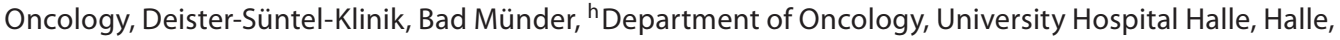
'Department of Oncology, University Hospital Regensburg, Regensburg, 'Oral and Maxillofacial Surgery, University Hospital Heidelberg, Heidelberg, and kDepartment of Oncology, University Hospital Würzburg, Würzburg, Germany
\end{abstract}

\section{Key Words}

Cetuximab - Disease control rate $\cdot$ Docetaxel $\cdot$ Platinum sensitivity · Squamous cell carcinoma of the head and neck

\footnotetext{
Abstract

Background: Cetuximab and docetaxel have single-agent activity in squamous cell carcinoma of the head and neck (SCCHN). The efficacy of their combination was evaluated in platinum-pretreated patients with recurrent and/or metastatic SCCHN. Patients and Methods: A total of 84 patients were treated with docetaxel $35 \mathrm{mg} / \mathrm{m}^{2}$ weekly for a maximum of 6 cycles and concomitant cetuximab $250 \mathrm{mg} / \mathrm{m}^{2}$ weekly until disease progression or unacceptable toxicity. The primary endpoint was the objective response rate and secondary endpoints included the response rate in relation
}

to platinum sensitivity, progression-free survival (PFS), overall survival (OS) and toxicity. Results: Nine (11\%) patients achieved a partial response and 34 (40\%) stable disease, resulting in a disease control rate of $51 \%$. Response to treatment was $49 \%$ in previously platinum-sensitive and $50 \%$ in previously platinum-resistant disease. The median PFS was 3.1 months and the median OS 6.7 months. The most common grade 3 or 4 adverse events were mucositis (8\%), pneumonia (8\%), fatigue (8\%) and skin reactions (14\%). Sepsis occurred in 3 patients. Conclusion: Cetuximab plus docetaxel is an active treatment regimen with moderate toxicity in SCCHN patients. However, no superiority in comparison with monotherapy could be shown. Responsiveness and survival were independent of previous platinum sensitivity.

Copyright $\odot 2013$ S. Karger AG, Basel

\section{KARGER}

E-Mail karger@karger.com www.karger.com/ocl
(C) 2013 S. Karger AG, Basel

0030-2414/13/0845-0284\$38.00/0
Prof. Dr. Ulrich Keilholz

Department of Hematology and Medical Oncology

Charité Campus Benjamin Franklin, Hindenburgdamm 30

DE-12200 Berlin (Germany)

E-Mail ulrich.keilholz@charite.de 


\section{Introduction}

Head and neck cancer is the 6th most common cancer in the world, with approximately 600,000 new cases per year. Most head and neck cancers, approximately $90 \%$ in Western societies, are squamous cell carcinomas (SCCHN). For patients with recurrent and/or metastatic disease the prognosis is very poor. Patients experiencing disease progression on platinum-based chemotherapy have a bleak prognosis, with a reported median survival of just about 3 months [1]. Patients with recurrent and/or metastatic disease without curative local treatment options are generally incurable, and currently available treatment options for recurrent and/or metastatic disease are limited. The aims of treatment include palliation of symptoms and prevention from new cancer-related-symptoms, progression-free survival (PFS) and overall survival (OS).

Chemotherapy is generally the treatment of choice in recurrent or metastatic disease. However, as results are very limited, there is a need for identification of new therapeutic strategies in both patients without previous treatment and in refractory patients. Recently, molecular-targeted therapies are new treatment options for SCCHN. The most extensively studied molecular mechanism of action in SCCHN is the epidermal growth factor receptor (EGFR) pathway. The receptor is uniformly (90-100\%) expressed in head and neck cancer cells. Cetuximab (Erbitux $^{\circledR}$ ) is an IgG1 monoclonal antibody that induces antibody-dependent cell-mediated cytotoxicity by inhibition of EGFR ligand binding [2-7]. Cetuximab has demonstrated single-agent activity in recurrent or metastatic SCCHN that progresses in spite of platinum-containing therapy. Chemotherapy has been combined with EGFR inhibitors as the two modalities differ in their mechanisms of action [8-12]. In recent years, taxanes have shown activity in SCCHN as single agent and as neoadjuvant and concomitant chemotherapy combination regimens. Weekly docetaxel at a dose of $30 \mathrm{mg} / \mathrm{m}^{2}$ was active in a phase II study in chemotherapy-naive recurrent and metastatic SCCHN with a reported response rate of $42 \%$ and a median survival of 11.3 months [13]. A phase II randomized trial showed higher response rates for weekly docetaxel compared to methotrexate in pretreated patients ( 27 vs. $15 \%$, respectively). However, the poor survival rates in both treatment arms warrant the search for more active regimens with acceptable toxicity [14]. Therefore, this study was designed to determine the efficacy and safety of the combination of weekly docetaxel plus cetuximab in platinum-pretreated patients with recurrent and/or metastatic SCCHN.

\section{Patients and Methods}

\section{Patient Selection}

Patients were eligible if they were 18 years of age or older and had histologically or cytologically confirmed incurable recurrent and/or metastatic SCCHN. Other inclusion criteria included prior platinum-containing chemotherapy; at least one lesion being measurable according to RECIST criteria 1.1, a Karnofsky performance score (KPS) $\geq 70 \%$, and adequate hematologic, renal and hepatic function. The main exclusion criteria were previous treatment with docetaxel or previous exposure to an EGFR pathwaytargeting agent and symptomatic peripheral neuropathy.

\section{Study Design}

This was an open-label, uncontrolled, interdisciplinary, multicenter, phase II study from the Arbeitsgemeinschaft Internistische Onkologie (AIO), which was conducted at 10 institutions in Germany. The primary endpoint was the objective response rate according to RECIST criteria. Secondary endpoints were response rate in relation to previous platinum sensitivity or platinum resistance, PFS (time from first dose of medication to the first confirmation of disease progression or the day of death as a result of any cause within 60 days after the last tumor assessment), OS (time from the first dose of medication to death) and safety. Adverse events were graded according to Common Toxicity Criteria (version 3.0). Platinum resistance was defined as disease progression within 3 months following the last platinum administration. The study was designed by the Head and Neck Committee of the AIO. Data were collected by the investigators at each center. The trial protocol and amendment were approved by the independent ethics committee of each center and by the authorities in Germany. The trial was conducted in accordance with the Declaration of Helsinki (1996). All patients provided written informed consent.

As an optional side study, blood and tissue samples were collected in patients who consented to blood collection in order to investigate potential prognostic factors. These investigations have been published elsewhere $[15,16]$.

\section{Treatment}

Patients received cetuximab at an initial dose of $400 \mathrm{mg} / \mathrm{m}^{2}$ body surface area given as a 2-hour intravenous infusion, followed by subsequent weekly doses of $250 \mathrm{mg} / \mathrm{m}^{2}$ as 1-hour intravenous infusions, ending at least $1 \mathrm{~h}$ before the start of chemotherapy. Docetaxel at a dose of $35 \mathrm{mg} / \mathrm{m}^{2}$ as a 1-hour intravenous infusion was administered on days 1,8 and 15 every 4 weeks. Dose modifications of cetuximab and docetaxel were permitted according to protocol-specified criteria. Patients received a maximum of 6 cycles (18 doses) of docetaxel in the absence of limiting toxicity or disease progression. After a maximum of 6 cycles of chemotherapy, patients who continued to have at least stable disease received further cetuximab maintenance treatment until disease progression or unacceptable toxicity.

\section{Assessment}

Tumor evaluation was assessed by computed tomography or magnetic resonance imaging at baseline and at 8 -week intervals until disease progression. The RECIST criteria were used to determine tumor response and disease progression. Blood samples (blood cell count, serum chemistry and electrolytes) were taken 
on a regularly basis for evaluations of toxicity. Concomitant medications and adverse events were monitored weekly throughout the study. Survival status and any further anticancer treatments were documented at follow-up visits every 3 months after disease progression.

\section{Statistical Analysis}

Continuous variables were summarized using descriptive statistics. Categorical variables were summarized using counts and percentages. Two-sided confidence intervals (CI) according to Clopper-Pearson were calculated for response rates. Kaplan-Meier estimates were used for time to event parameters, including PFS and OS. The response rates according to platinum sensitivity versus platinum resistance were compared using Fisher's exact test. Cox's proportional hazard model was used for multivariate analysis to identify independent prognostic factors for survival. The primary population for efficacy analyses was the intent-to-treat population (defined as all patients enrolled into the study). Safety analyses were conducted on the safety analysis set, defined as all patients enrolled into the study who received at least one dose of cetuximab and docetaxel. The trial initially had a two-stage design: if $\leq 1$ response was observed in the first 18 patients, the study had to be stopped due to futility. Otherwise accrual was to be continued to a total of 47 patients, and if the total number of responses were to exceed 4, cetuximab in combination with docetaxel should be further investigated. For more robust determination of the secondary endpoint PFS, an amendment to the protocol was instituted to increase the accrual to 84 patients and provide an estimate of PFS suitable for planning future randomized trials.

\section{Results}

A total of 84 patients were enrolled at 10 institutions in Germany within 17 months between December 2006 and April 2008. All patients were included in the intentto-treat population. The safety population comprised 84 patients. Nine patients who received 6 cycles of chemotherapy continued treatment with cetuximab maintenance.

\section{Patient Characteristics}

Demographic and clinical baseline characteristics are listed in table 1 . The patient population comprised 69 males with a median age of 59 years. Baseline KPS was good (KPS $90-100 \%$ ) in 19\% of the patients. The most common site of the primary tumor was the oropharynx (33\%; table 1). Distant metastases were present in 39\% of patients. The median number of previous treatments including operation, radiotherapy and chemotherapy was 2 (range, 1-9). The disease was regarded as platinum sensitive in $56 \%$ of the patients, platinum resistant in $26 \%$ of the patients and not evaluable in further $18 \%$ of the patients due to lack of data.
Table 1. Demographic and clinical characteristics of the patients at baseline

\begin{tabular}{lc}
\hline Characteristics & Patients, $\mathrm{n}(\%)$ \\
\hline Total cohort & 84 \\
Males & $69(82)$ \\
KPS & $16(19)$ \\
$90-100 \%$ & $63(75)$ \\
$70-80 \%$ & $5(6)$ \\
Not evaluable & \\
Primary tumor site & $12(14)$ \\
$\quad$ Hypopharynx & $4(5)$ \\
Larynx & $28(33)$ \\
Oropharynx & $16(19)$ \\
Oral cavity & $14(17)$ \\
Other & $10(12)$ \\
$\quad$ Not evaluable & \\
Extent of disease at inclusion & $33(39)$ \\
Metastatic & $18(21)$ \\
Recurrent & $17(20)$ \\
Recurrent and metastatic & $16(20)$ \\
Not evaluable & \\
Response to platinum-based therapy & $47(56)$ \\
Platinum sensitive & $22(26)$ \\
Platinum resistant & $15(18)$ \\
Not evaluable &
\end{tabular}

Median age of the patients was 59 years (range, 34-79).

\section{Exposure to Treatment}

The median duration of treatment was 2.9 months (range, $0-17.7$ ). Patients received a median number of 3.3 cycles with docetaxel and a median number of 3.7 cycles with cetuximab. Nine patients (11\%) received cetuximab monotherapy as the maintenance treatment, with a median duration of 11.6 months.

\section{Treatment Efficacy}

According to the RECIST criteria, the overall response and disease control rate (DCR) included 9 partial responses and 34 patients with stable disease, respectively, resulting in an overall response rate of $11 \%$ and a DCR of $51 \%$ (table 2). No patient achieved a complete response. There were, additionally, 3 short-lived responses after 2 cycles that were not confirmed, because of progression after 4 cycles. The overall median duration of response was 126 days. Protocol subgroup analyses showed that the overall responses to the study treatment of platinum-sensitive and -resistant patients were very similar in both subgroups: 11 of the 22 platinum-resistant patients experienced disease control (DCR 50\%) and 23 of the 47 platinum-sensitive pa- 
Fig. 1. Kaplan-Meier estimates of survival.

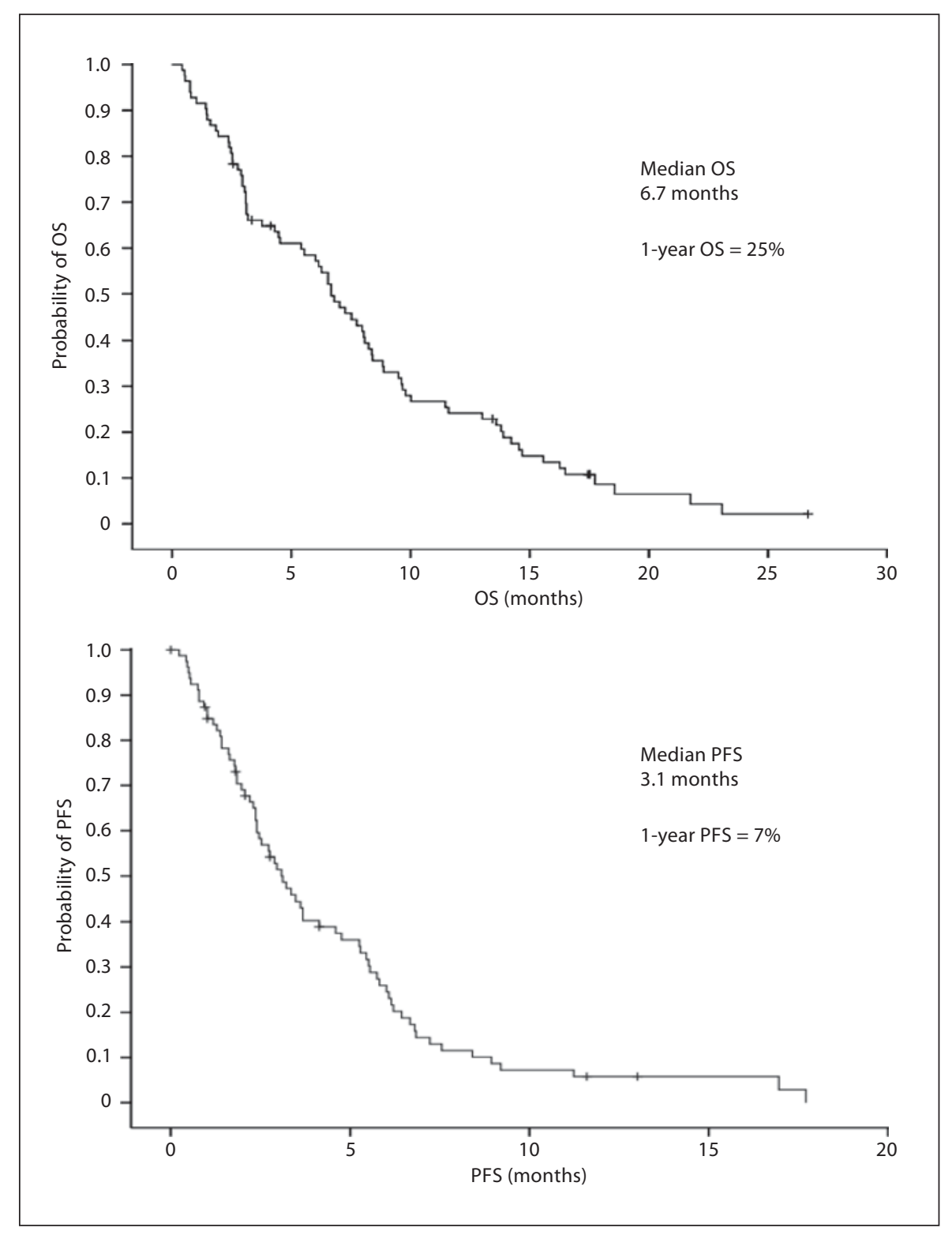

tients (DCR 49\%). Previous platinum sensitivity was not predictive for PFS $(\mathrm{p}=0.4)$ and OS $(\mathrm{p}=0.94)$.

\section{Time to Progression, PFS and OS}

The median PFS was 3.1 months (95\% CI, 2.3-3.9; fig. 1) and the proportion of patients without progression was $21 \%$ at 6 months and $7 \%$ at 1 year. The median OS was 6.7 months (95\% CI, 5.3-8.0; fig. 1) with $58 \%$ of patients alive at 6 months and $25 \%$ at 1 year. Among the patients receiving cetuximab maintenance after completion of the combination period, the median further PFS was 18 weeks from the start of maintenance treatment.
Table 2. Response to treatment

\begin{tabular}{lc}
\hline Response variable & Patients, $\mathrm{n}(\%)$ \\
\hline Best response & \\
Partial response & $9(11)$ \\
Stable disease & $34(40)$ \\
Progressive disease & $36(43)$ \\
Not evaluable & $5(6)$ \\
DCR & $43(51)$ \\
\hline
\end{tabular}


Table 3. Grade $3 / 4$ adverse events

\begin{tabular}{lrr}
\hline Adverse events & $\mathrm{n}$ & $\%$ \\
\hline Skin reactions & 12 & 14 \\
Mucositis & 7 & 8 \\
Pneumonia & 7 & 8 \\
Fatigue & 7 & 8 \\
Anemia & 4 & 5 \\
Sepsis & 3 & 4 \\
Tumor hemorrhage & 3 & 4 \\
Decreased performance status & 3 & 4 \\
Dyspnea & 3 & 4 \\
Headache & 2 & 2 \\
Febrile neutropenia & 2 & 2 \\
Infusion-related reactions & 2 & 2 \\
Cardiac events & 1 & 1 \\
Perforation of a duodenal ulcer & 1 & 1 \\
\hline
\end{tabular}

Multivariate analysis did not reveal a prognostic factor for survival, considering the following variables: platinum sensitivity, age, sex, extent of disease (locally recurrent/metastatic), primary tumor site, and exposure to tobacco or alcohol.

\section{Safety}

The safety profile was consistent with the anticipated toxicity for both agents used. The most common hematologic adverse event of grade 3 and 4 was anemia in 5\% of the patients. The most frequent non-hematologic adverse events were skin reactions, mucositis, pneumonia and fatigue. Skin reactions included rash, acne, nail disorders and dry skin. Two patients had severe infusionrelated reactions. Sepsis occurred in 3 patients and febrile neutropenia in 2 patients. Adverse events of grade 3 and 4 were observed in $67 \%$ of the patients (table 3 ). Serious adverse events, mostly grade 3 or 4 , were reported in 46 patients (55\%). Four patients died during the chemotherapy phase for reasons not related to tumor progression. Causes of death in these 4 patients included sudden death, catheter port infection and subsequent pneumonia, PEG tube infection and subsequent pneumonia during neutropenia, and sepsis from superinfected tumor.

\section{Discussion}

Locoregional or distant relapses occur in approximately half of the SCCHN patients, and treatment remains unsatisfactory in patients with relapse after plati- num-containing treatment. At present, no standard regimen is available in this setting. Several phase II trials have examined numerous systemic treatment options mostly in first-line treatment with several cytotoxic agents, including docetaxel and also cetuximab monotherapy, with overall response rates between 10 and $20 \%$ and a median survival of 6-9 months [10-13]. Here we report clinical activity of the combination of docetaxel with cetuximab in second-line systemic treatment. Given the unfavorable patient characteristics of our cohort, our results compare well with other treatment regimens investigated in this disease. The results of this study compare well to those for single-agent docetaxel in recurrent and/or metastatic SCCHN with overall response rates between 6.7 and 13\%, with a corresponding DCR of $33-34 \%$ in several studies. However, in several studies, response rates were higher than in our study, irrespective of whether a weekly or a 3 -weekly regimen was used [13, 14, 17]. Median PFS and OS of 7.4-9 and 17.9-29 weeks, respectively, have been reported previously $[14,17,18]$. The survival data were remarkably similar to OS and PFS in our study. These differences could be potentially explained by differences in the study population as most of these studies included chemonaive patients or patients with a treatment-free interval of more than 6 or 12 months since the end of their initial treatment, or who had not received prior chemotherapy for recurrent and/or metastatic disease. Similar response rates with an overall response rate of $13 \%$ and about 6 months of OS were reported by cetuximab monotherapy [8]. Compared to the combination of cetuximab plus docetaxel, cetuximab as single agent has a mild toxicity profile. In both settings, docetaxel monotherapy or in combination with cetuximab, moderate toxicities were seen. The adverse events observed in our study were those expected with the agents used. Mucositis is commonly observed with docetaxel. Grade 3/4 toxicities were reported in $8 \%$ of the patients. Grade $3 / 4$ pneumonia was also observed in $8 \%$ of the patients, a percentage not reported in other trials. The discrepancy may be due to differences in patient selection. It is worth noting that in our study only pretreated and mostly preradiated patients were included. All patients have been heavy smokers with pulmonary comorbidities. In contrast to observations with cetuximab therapy, the incidence of grade $3 / 4$ skin toxicities $(14 \%)$ is low. No discrepancy in hematological toxicities was seen compared to docetaxel monotherapy.

One of the most unfavorable factors in many cancers is resistance to platinum treatment. In several other cancer types, namely ovarian cancer, platinum-refractory disease has been defined as progression within 6 months 
after completion of platinum-containing chemotherapy. A similar definition has not been employed for SCCHN; however, most clinical trials have excluded patients with progression within an interval of at least 6 months after prior cisplatin exposure, whereas $27 \%$ of our patients entered the study after progression on or briefly after prior cisplatin treatment. Unexpectedly, the efficacy of our study treatment appeared to be independent of prior platinum sensitivity, which suggests that there is no relevant cross-resistance of the two approaches. The consequence of the latter observation is twofold: on the one hand, the combination of docetaxel and cetuximab is an active treatment option for patients with recurrent and/or metastatic SCCHN regardless of prior treatment, but on the other hand, the three agents cisplatin, docetaxel and cetuximab appear to be potential candidates for combina- tion first-line treatment or induction therapy based on the apparent lack of overt cross-resistance. This is currently being investigated in a first-line, randomized, phase II trial conducted by the AIO Head and Neck Cancer Group.

In conclusion, the results of our study are in line with single-agent docetaxel and with those of cetuximab monotherapy in more selected patient populations with metastatic and/or recurrent disease and warrant further investigation in randomized trials.

\section{Acknowledgment}

The study was supported by unrestricted educational grants from Merck-Serono KGaA and Sanofi-Aventis.

\section{References}

1 León X, Hitt R, Constenla M, Rocca A, Stupp R, Kovács AF, Amellal N, Bessa EH, Bourhis $\mathrm{J}$ : A retrospective analysis of the outcome of patients with recurrent and/or metastatic squamous cell carcinoma of the head and neck refractory to a platinum-based chemotherapy. Clin Oncol (R Coll Radiol) 2005;17: 418-424.

- 2 Goldstein NI, Prewett M, Zuklys K, Rockwell P, Mendelsohn J: Biological efficacy of a chimeric antibody to the epidermal growth factor receptor in a human tumor xenograft model. Clin Cancer Res 1995;1:1311-1318.

- 3 Li S, Schmitz KR, Jeffrey PD, Wiltzius JJ, Kussie P, Ferguson KM: Structural basis for inhibition of the epidermal growth factor receptor by cetuximab. Cancer Cell 2005;7: 301-311.

-4 Sato JD, Kawamoto T, Le AD, Mendelsohn J, Polikoff J, Sato GH: Biological effects in vitro of monoclonal antibodies to human epidermal growth factor receptors. Mol Biol Med 1983;1:511-529.

5 Kang X, Patel D, Ng S, Melchoir M, Ludwig D, Hicklin D: High affinity Fc receptor binding and potent induction of antibody-dependent cellular cytotoxicity (ADCC) in vitro by anti-epidermal growth factor receptor antibody cetuximab. J Clin Oncol 2007;25(sup$\mathrm{pl}): 128$.

- 6 Kimura H, Sakai K, Arao T, Shimoyama T, Tamura T, Nishio K: Antibody-dependent cellular cytotoxicity of cetuximab against tumor cells with wild-type or mutant epidermal growth factor receptor. Cancer Sci 2007; 98:1275-1280.

7 Zhang W, Gordon M, Schultheis AM, et al: FCGR2A and FCGR3A polymorphisms associated with clinical outcome of epidermal growth factor receptor expressing metastatic colorectal cancer patients treated with single-agent cetuximab. J Clin Oncol 2007;25: 3712-3718

-8 Vermorken JB, Mesia R, Rivera F, Remenar E, Kawecki A, Rottey S, Erfan J, Zabolotnyy D, Kienzer HR, Cupissol D, Peyrade F, Benasso M, Vynnychenko I, De Raucourt D, Bokemeyer C, Schueler A, Amellal N, Hitt R: Platinum-based chemotherapy plus cetuximab in head and neck cancer. N Engl J Med 2008;359:1116-1127.

-9 Vermorken JB, Trigo J, Hitt R, et al: Openlabel, uncontrolled, multicenter phase II study to evaluate the efficacy and toxicity of cetuximab as a single agent in patients with recurrent and/or metastatic squamous cell carcinoma of the head and neck who failed to respond to platinum-based therapy. J Clin Oncol 2007;25:2171-2177.

10 Baselga J, Trigo JM, Bourhis J, et al: Phase 11 multicenter study of the antiepidermal growth factor receptor monoclonal antibody cetuximab in combination with platinumbased chemotherapy in patients with platinum-refractory metastatic and/or recurrent squamous cell carcinoma of the head and neck. J Clin Oncol 2005;23:5568-5577.

11 Herbst RS, Arquette M, Shin DM, et al: Phase II multicenter study of the epidermal growth factor receptor antibody cetuximab and cisplatin for recurrent and refractory squamous cell carcinoma of the head and neck. J Clin Oncol 2005;23:5578-5587.

12 Vermorken JB, Herbst RS, Leon X, Amellal $\mathrm{N}$, Baselga J: Overview of the efficacy of cetuximab in recurrent and/or metastatic squamous cell carcinoma of the head and neck in patients who previously failed platinum-based therapies. Cancer 2008; 112: 2710-2719.
13 Hitt R, Amador ML, Quintela-Fandino M, Jimeno A, del Val O, Hernando S, et al: Weekly docetaxel in patients with recurrent and/or metastatic squamous cell carcinoma of the head and neck. Cancer 2006;106:106111.

14 Guardiola E, Peyrade F, Chaigneau L, Cupissol D, Tchiknavorian X, Bompas E, et al: Results of a randomised phase II study comparing docetaxel with methotrexate in patients with recurrent head and neck cancer. Eur J Cancer 2004;40:2071-2076.

15 Klinghammer K, Knödler M, Schmittel A, Budach V, Keilholz U, Tinhofer I: Association of epidermal growth factor receptor polymorphism, skin toxicity, and outcome in patients with squamous cell carcinoma of the head and neck receiving cetuximabdocetaxel treatment. Clin Cancer Res 2010; 16:304-310.

16 Tinhofer I, Klinghammer K, Weichert W, Knödler M, Stenzinger A, Gauler T, Budach V, Keilholz U: Expression of amphiregulin and EGFRvIII affect outcome of patients with squamous cell carcinoma of the head and neck receiving cetuximab-docetaxel treatment. Clin Cancer Res 2011;17:51975204.

$\checkmark 17$ Cho BC, Keum KC, Shin SJ, Choi HJ, Lee YJ, KIM SH, Choi EC, Kim JH: Weekly docetaxel in patients with platinum-refractory metastatic or recurrent squamous cell carcinoma of the head and neck. Cancer Chemother Pharmacol 2009;65:27-32.

18 Specenier P, Rasschaert M, Vroman P, Van den Brande J, Dyck J, Schrijvers D, Huizing MT, Vermorken JB: Weekly docetaxel in patients with recurrent and/or metastatic squamous cell carcinoma of the head and neck. Am J Clin Oncol Epub 2011;34:472-427. 